\title{
Prisoners' Dilemma Supergame on Rectangle Lattice
}

\author{
Zhongxing Ye $\mathrm{e}^{1,2}$, Jingshu Chen ${ }^{3}$ \\ ${ }^{1}$ School of Business Information Management, Shanghai Institute of Foreign Trade, Shanghai, China \\ ${ }^{2}$ Department of Mathematics, Shanghai Jiao Tong University, Shanghai, China \\ ${ }^{3}$ Department of Management Science and Engineering, Stanford University, Stanford, USA \\ Email: yezx@shift.edu.cn,jingshuc@stanford.edu
}

Received 2013

\begin{abstract}
In this paper a class of large supergames, i.e., infinitely repeated games played by many players are studied. The players located on the vertex set of planar rectangle lattice play several basic games with his neighbors. The basic game is two-person prisoners' dilemma game with asymmetric payoffs. Under the conditions of the pre-specified updating rules and the transition probabilities, the relevant stochastic process of strategy evolution forms a Markovian process. The simulation results about the long-run behavior are provided.
\end{abstract}

Keywords: Prisoners' Dilemma; Supergame; Planar Rectangle Lattice; Markov Process; Invariant Measure; Equilibrium

\section{Introduction}

In a series of our previous works we have investigated a class of large Ising-type supergames, i.e., infinitely repeated games played by (infinitely) many players located on networks. In these stylized class of supergames, game players are located on the vertex set of planar rectangle lattice or trees (see [1,2]). Each player plays several basic games with his neighbors. The basic game is two-person Ising-type game with symmetric payoffs. Under the conditions of the pre-specified updating rules and the transition probabilities i.e., these relevant stochastic process of strategy configuration given, the formula of invariant measures which represent the long-run equilibrium plays are obtained. The phase transition phenomena are discovered.

In this work we extend our previous work to prisoners' dilemma game with asymmetric payoffs in stead of Ising-type game with symmetric payoffs. There has been many research works on prisoner's dilemma games (see [3]) and for evolutionary prisoner's dilemma games (for example, see [4,5]). In this work, we assume that each player plays several two person prisoners' dilemma games only with her neighbors, in each and every period of discrete times. Players change their strategy simultaneously at every period of time. In section 2 , we offer the structures of planar rectangle lattices and the formulation of the class of prisoners' dilemma supergames by offering the ingredients needed. In section 3 , we study a special dynamic supergame with basic two person prisoners' dilemma game with asymmetric payoffs. The theoretic analysis is difficult. So we provide simulated results to pursue the limiting behavior of the dynamics. Section 4 is the conclusion, in which some further research directions are mentioned.

\section{Supergames on Planar Rectangle Lattices Based on Basic Two Person Prisoners' Dilemma Games}

The players: We assume that players are located on the vertex sites of a planar rectangle lattice (see Figure 1) $G=(V, E)$, where $V$ is the vertex set and $E$ the edge set of the lattice. We also assume that all the players are identical.

Neighborhood: A neighborhood system $N=\left\{N_{i}, i \in V\right\}$ is a collection of nonempty subsets of the vertices of $V$ such that (i) $i$ does not belong to $N_{i}$ for all $i \in V$; (ii) $i \in N_{j}$ if and only if $j \in N_{i}$, for all $i, j \in V ; N_{i}$ is called the neighborhood of $i$. We define the set $W_{i}=N_{i} \cap\{i\}$. In this work we only consider the following structure of neighborhood. The structure of neighboring sites of the origin $o=(0,0)$ is given by

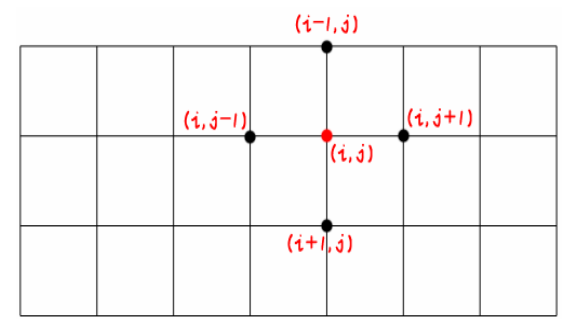

Figure 1. Planar rectangle lattice and neighborhood structure. 


$$
N_{o}=\{(1,0),(-1,0),(0,1),(0,-1)\}
$$

(see Figure 1). This means that each vertex has 4 nearest neighboring vertices.

Prisoners' dilemma game: The basic game we study in this work is two person prisoners' dilemma game (PDG) which is defined in its classic form: This game is played by two players. We assume that each player has only two choices of strategies which may be identified as $A=\{C, D\}$. Where $C$ represents to cooperate and $D$ represents defect. At any run of dynamic games, if both players choose $C$, they get a pay-off $R$ each; if one player chooses $D$ while the other chooses $C$, the defector player gets the biggest pay-off $T$, while the other gets $S$; if both players defect, they get pay-off $P$. We can write the payoff in the matrix form:

$$
\boldsymbol{Q}=\left(\begin{array}{ll}
Q(C, C) & Q(C, D) \\
Q(D, C) & Q(D, D)
\end{array}\right)=\left(\begin{array}{cc}
(R, R) & (S, T) \\
(T, S) & (P, P)
\end{array}\right)
$$

where the pay-off values must satisfy the inequalities

$$
T>R>P>S \text { and } 2 R>S+T \text {. }
$$

For more about PDG, readers may refer [3-5].

Stage games: In our class of supergames, some stage games are played over discrete time $i \in\{0,1,2, \cdots\}$. At each discrete time every player plays four 2-strategy 2person prisoners' dilemma games simultaneously with his neighbors. At the end of each game, player $i$ receives payoff $Q_{i j}\left(y, x_{j}\right)$ if he plays strategy $y$ while his neighbor $j$ plays strategy $x_{j}$; so his total payoff from playing strategy $y$ is the sum of the payoffs received from playing $y$ against each of his neighbors. Then player $i$ may revise his strategy from $y$ to $z$ with probability

$$
\begin{aligned}
& p_{i}(z \mid \boldsymbol{x}(i, y)) \\
= & \frac{1}{\lambda} \exp \left\{\beta \frac{1}{\left\|N_{i}\right\|} \sum_{j \in N_{i}}\left[Q_{i j}\left(z, x_{j}\right)-Q_{i j}\left(y, x_{j}\right)\right]\right\} \\
= & \frac{1}{\lambda^{\prime}} \exp \left\{\beta \frac{1}{\left\|N_{i}\right\|} \sum_{j \in N_{i}} Q_{i j}\left(z, x_{j}\right)\right\}
\end{aligned}
$$

where $\lambda$ and $\lambda^{\prime}$ are normalization factors to make

$$
\sum_{z \in A} p_{i}(z \mid \boldsymbol{x}(i, y))=1
$$

The global updating rule is synchronous, i.e., all players change their strategies simultaneously at the same time.

The dynamics of a supergame is characterized by a stochastic process which is called strategy evolution process (SEP). Technically, the SEP for a large supergame is a Markov chain whose state at time $\mathrm{t}$ is denoted by $\boldsymbol{X}_{t}=\left\{X_{t, i} ; i \in V\right\}$. It takes value over

$$
\Omega_{t}=\Omega=A^{V}=\{C, D\}^{V}
$$

which is called configuration space of the SEP at time t. $\boldsymbol{x}_{t}=\left\{x_{t, i} ; i \in V\right\}$ is the realization of $\boldsymbol{X}_{t}$. Equivalently we may model the state of SEP at time $t$ by a probability distribution $\mu_{t}$ on $A^{V}$. Suppose that the configuration $\boldsymbol{x}_{t-1}$ determines the strategy of player $i$ at time $t$ with probability (called local transition probability):

$$
p_{i}\left(x_{t, i} \mid \boldsymbol{x}_{t-1}\right)=p_{i}\left(x_{t, i} \mid x_{t-1, j}: j \in W_{i}\right\}
$$

Note that

$$
\sum_{x_{t, i}} p_{i}\left(x_{t, i} \mid x_{t-1, j} ; j \in W_{i}\right)=1 \text { for all } i \in V
$$

Let $P(\boldsymbol{y} \mid \boldsymbol{x})$ be the global one-step transition probabilities from $\boldsymbol{x}$ to $\boldsymbol{y}$. Then for Synchronous updating rule, the global transition probabilities of the SEP are defined by

$$
P\left(\boldsymbol{x}_{t} \mid \boldsymbol{x}_{t-1}\right)=\prod_{i \in V} p_{i}\left(x_{t, i} \mid x_{t-1, j}: j \in W_{i}\right)
$$

The global transition probabilities (6) defines a discrete-time Markov process on the configuration space $A^{V}$. Given a measure $\rho_{t-1}$ on the configuration $\boldsymbol{x}_{t-1}$ (6) defines a probability measure $\rho_{t}=\rho_{t-1} P$ on $\boldsymbol{x}_{t}$.

$$
\rho_{t}\left(d \boldsymbol{x}_{t}\right)=\int \rho_{t-1}\left(d \boldsymbol{x}_{t-1}\right) P\left(d \boldsymbol{x}_{t} \mid \boldsymbol{x}_{t-1}\right)
$$

We say that a measure $v$ is stationary or time invariant if $v=v P$. We are interested in existence and uniqueness of the invariant measures under the above mentioned condition, i.e., the ergodicity and reversibility of the SEP. In certain cases there may exist multiple invariant measures. This phenomenon is called phase transition. The following result is well known.

Theorem 3.1: The invariant measures for the time evolution form a nonempty convex set.

Proof: see [6].

For the synchronous updating case, to find the invariant measure analytically is quite difficult. So in this paper we focus on the numerical simulation which shows interesting behavior. The detail is given in the next section.

\section{Simulation Result of the Limiting Behavior}

In this section we report the simulation results for examining the limiting behavior of dynamic supergames described in the previous section. For convenience, we set the following payoff matrix

$$
Q=\left(\begin{array}{cc}
(-1,-1) & (-8,0) \\
(0,-8) & (-5,-5)
\end{array}\right)
$$

We consider the finite sub-lattice with $40 \times 40$ vertices and 3 different boundary conditions. The local and global transition probabilities are given by (2) and (6), respectively. We use white color to represent $D$ strategy state, and black color the $C$ strategy state. For different 
value of parameter $\beta$, we simulate the dynamics of evolution 500 steps. For simplicity, we only report the following three cases. The dynamic percentage of write vertices and the final state are provided via graphs.

1) Case 1. $\beta=0$

The change of every player's strategy is independent of the history of his own and his neighbors' past strategies. So this case is not of interest.

2) Case 2. $\beta=1$

i) The white boundary conditions: (Figure 2)

ii) The black boundary conditions: (Figure 3)

iii) The periodic boundary conditions: (Figure 4)

3) Case 3. $\beta=3$

i) White boundary conditions: (Figure 5)

ii) Black boundary conditions: (Figure 6)

iii) Periodic boundary conditions: (Figure 7)

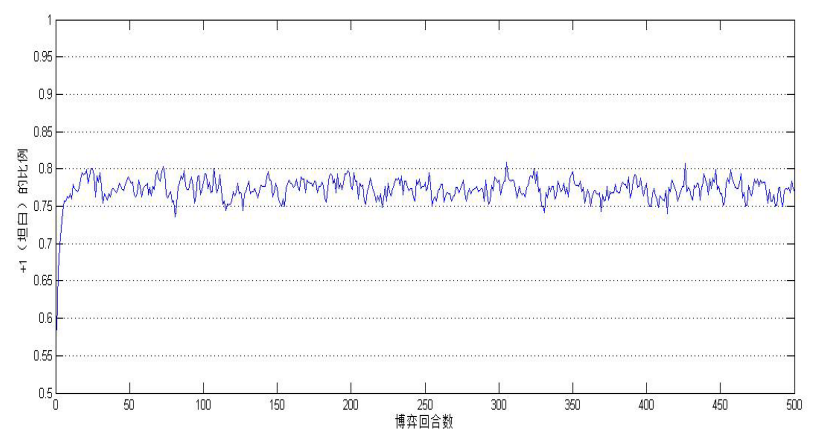

(a)

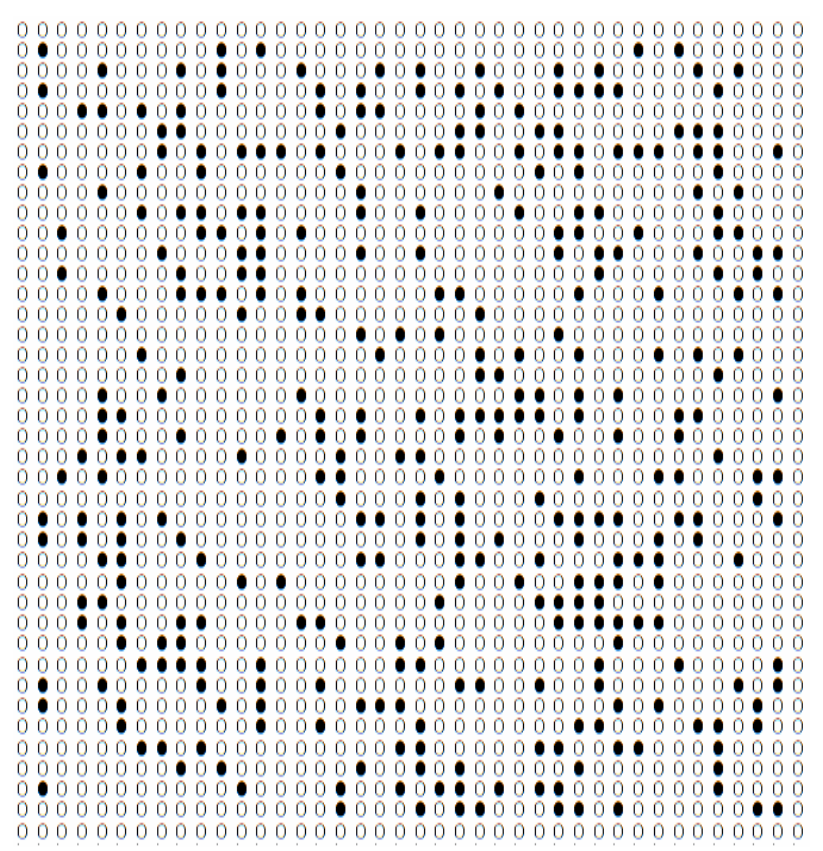

(b)

Figure 2. (a) $\beta=1$ case with white boundary conditions: the dynamic percentage of write vertices; (b) $\beta=1$ case with white boundary conditions: the final configuration after 500 steps of evolution.

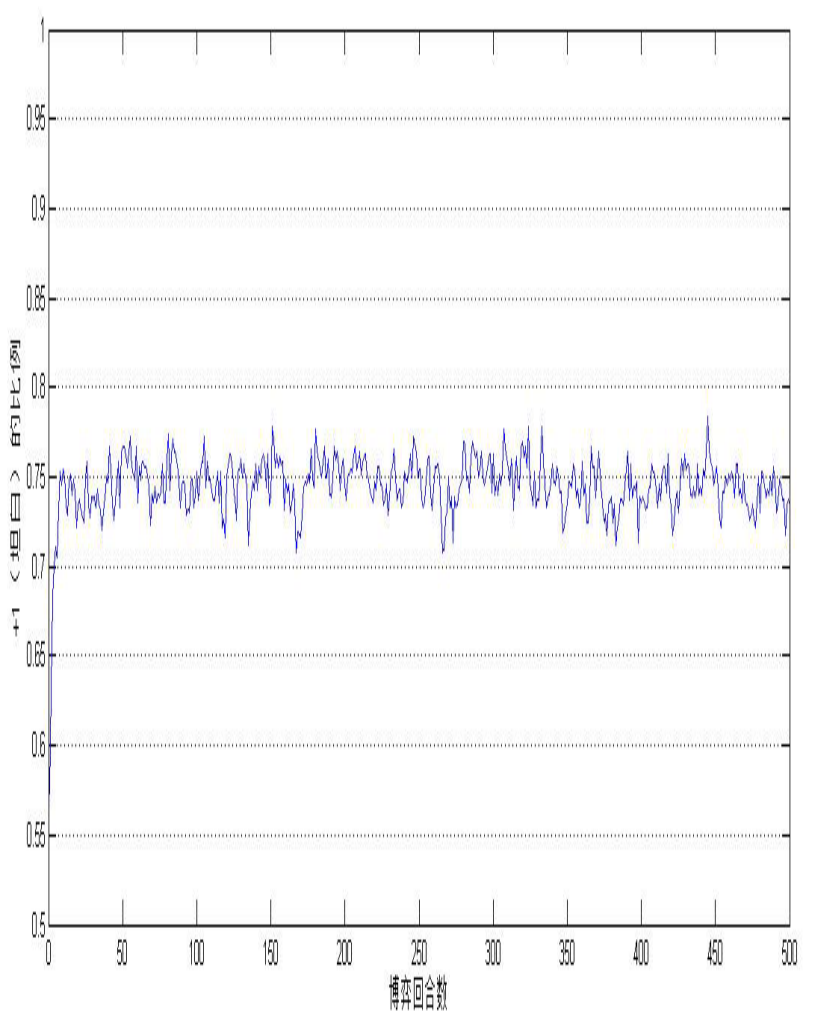

(a)

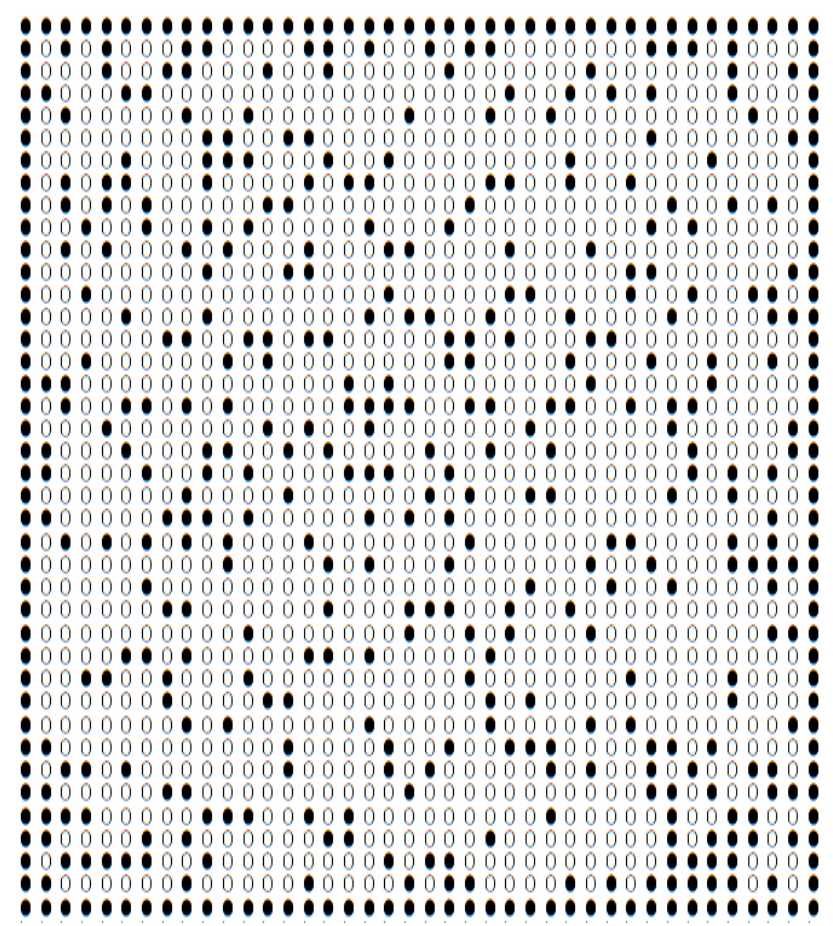

(b)

Figure 3. (a) $\beta=1$ case with black boundary conditions: the dynamic percentage of write vertices; (b) $\beta=1$ case with black boundary conditions: the final configuration after 500 steps of evolution. 


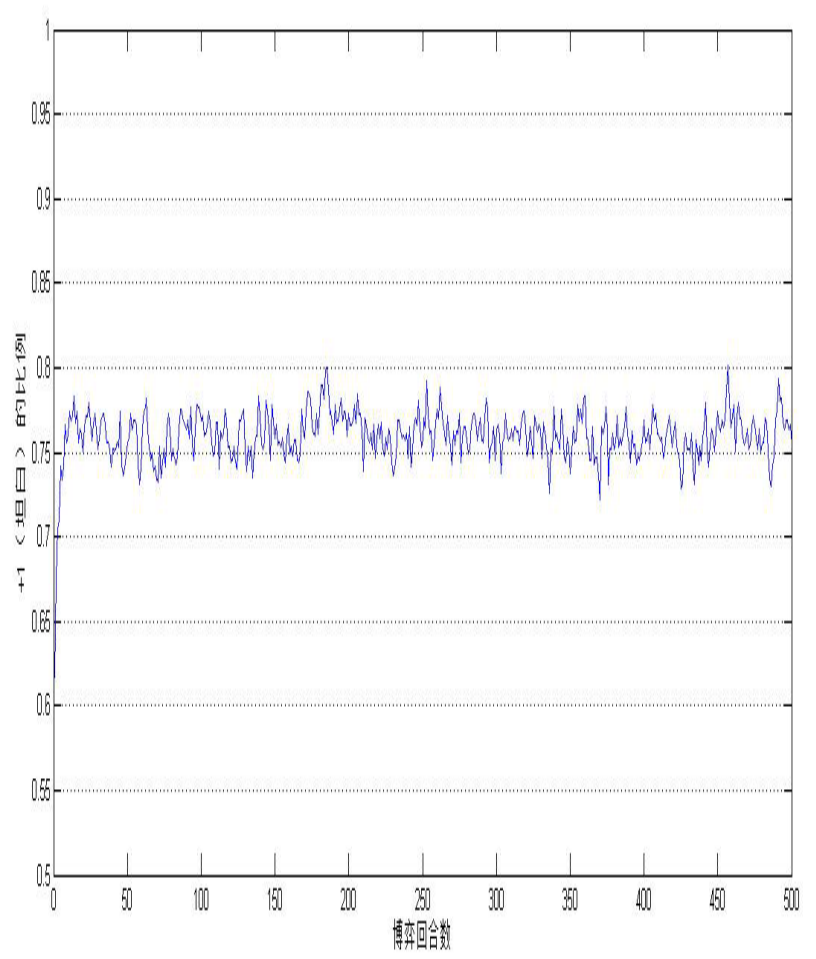

(a)

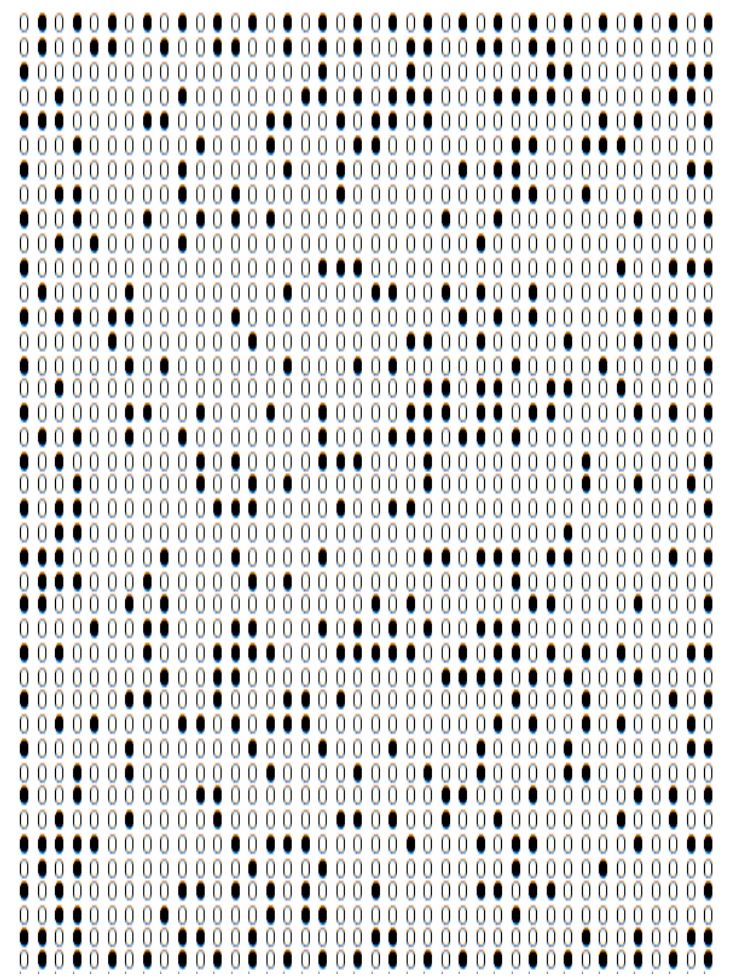

(b)

Figure 4. (a) $\beta=1$ case with periodic boundary conditions: the dynamic percentage of write vertices; (b) $\beta=1$ case with periodic boundary conditions: the final configuration after 500 steps of evolution.

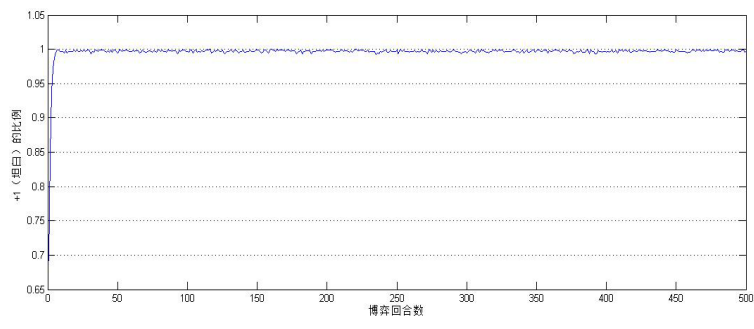

(a)

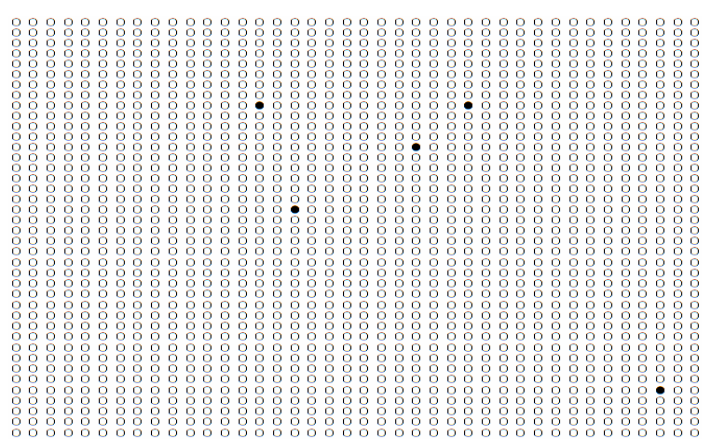

(b)

Figure 5. (a) $\beta=3$ case with white boundary conditions: the dynamic percentage of write vertices; (b) $\beta=3$ case with white boundary conditions: the final configuration after 500 steps of evolution.

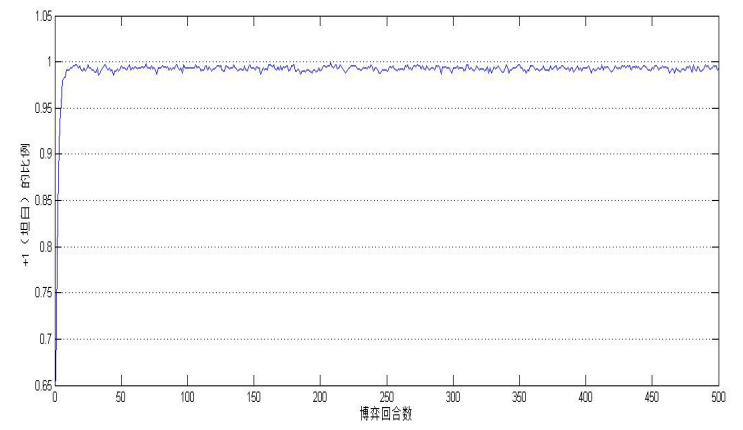

(a)

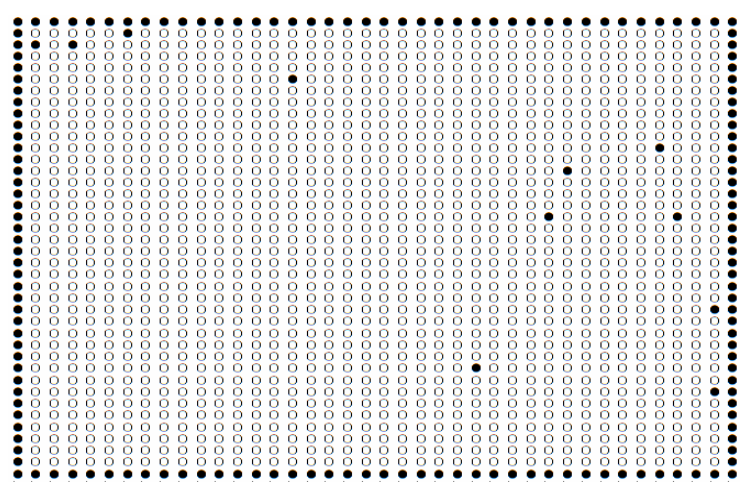

(b)

Figure 6. (a) $\beta=3$ case with black boundary conditions: the dynamic percentage of write vertices; (b) $\beta=3$ case with black boundary conditions: the final configuration after 500 steps of evolution. 
From the above simulation result, we can see that for small value of $\beta(\beta>0)$, the dynamic percentage of white vertices approaches to $50 \%$, while for large value of $\beta \quad(\beta>0)$ the dynamic percentage of white vertices approaches to $100 \%$. In other word, the final configuration of SEP will approach to the sole state with all white color. This means that all players approach to defect. The greater the $\beta$, the faster the convergence. So we conjecture that there exists a critical value of $\beta$ denoted by $\beta_{c}$ such that for $0<\beta<\beta_{c}$ and $\beta>\beta_{c}$ the limiting behavior of the dynamic supergames are different. For $0<\beta<\beta_{c}$ cases, the dynamic percentages of white vertices approache to $50 \%$. While for $\beta>\beta_{c}$ cases, the dynamic percentages of white vertices approache to $100 \%$. To find the exact value of $\beta_{c}$ is also of interest. But the problem to prove these confirmations and conjecture theoretically remains open.

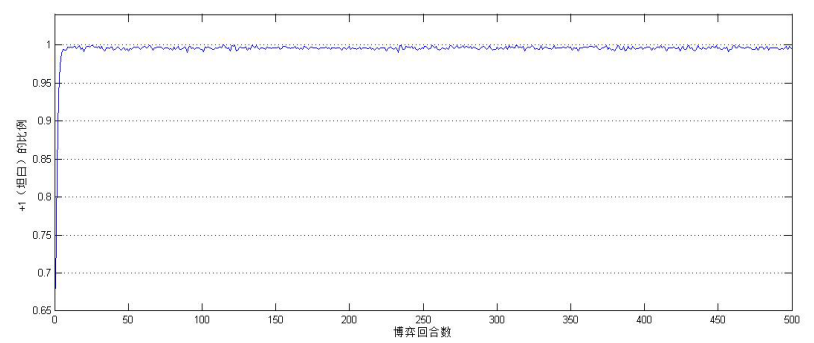

(a)

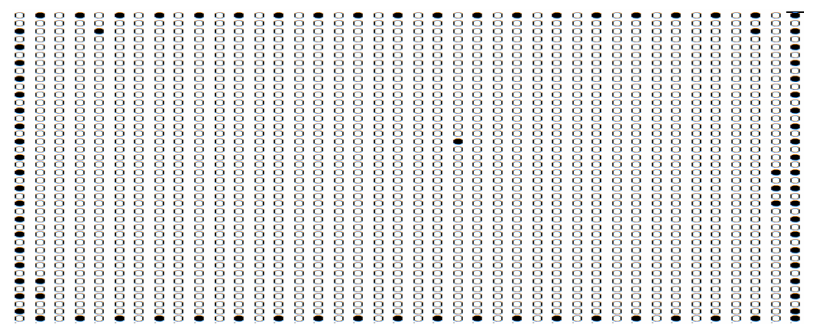

(b)

Figure 7. (a) $\beta=3$ case with periodic boundary conditions: the dynamic percentage of write vertices; (b) $\beta=3$ case with periodic boundary conditions: the final configuration after 500 steps of evolution.

\section{Conclusions}

We study the dynamic prisoners' dilemma supergame on rectangle lattice and provide some interesting simulation results and some conjucture. The theoretic analysis remains open. Other types of supergames on different lattices and based on other basic games will be reported elsewhere.

\section{Acknowledgements}

The authors would like to thank the National Natural Science Foundation of China (Program No. 11171215) and Shanghai 085 Project for financial supports.

\section{REFERENCES}

[1] A. J. Wang, Z. X. Ye and Y. C. Liu, "On the Long-run Equilibria of a Class of Large Super Game on $\mathrm{Z}^{\wedge} \mathrm{d}$," Journal of Mathematical Sciences: Advances and Applications, Vol. 12, No. 1, 2012, pp. 21-46.

[2] H. Ou and Z. X. Ye, "Dynamic Supergames on Trees," Proceeding of 2010 International Conference on Progress in Informatics and Computing(PIC-2010), Shanghai, December 10-12, 2010.

[3] G. Kendall, X. Yao, S. Y. Chong, "The Iterated Prison ers' Dilemma: 20 Years On," World Scientific Publishing Co Pte Ltd, 2007.

[4] Y. K. Liu, Z. Li, X. J. Chen and L. Wang, "Evolutionary Prisoners' Dilemma Game on Highly Clustered Community Networks," Chinese Physics, Vol. 18, No. 7, 2009, pp. 2623-2628. doi: 10.1088/1674-1056/18/7/001

[5] M. A. Saif and P. M. Gade, "Prisoner's Dilemma with Semi- synchronous Updates: Evidence for a First Order Phase Transition," Vol. 6, 2009.

[6] R. Kinderman and J. L. Snell, "Markov Random Fields and Their Applicarions," American Mathematical Society (AMS), Providence Rhode Island, 1980._doi: 10.1090/conm/001 